\title{
MIDDLE EAR AND NASOPHARYNGEAL BACTERIAL FLORA AND THEIR SENSITIVITY IN ACTIVE STAGE OF MUCOSAL DISEASE OTITIS MEDIA: A COMPARISON
}

\section{RAKESH VUPPALA ${ }^{1 *}$, KATHYAYANI BURUGULA ${ }^{2}$, BALAKRISHNAN R ${ }^{3}$}

${ }^{1}$ Department of ENT, Apollo Institute of Medical Sciences and Research, Hyderabad, Telangana, India. ${ }^{2}$ Department of Otorhinolaryngology, Kamineni Academy of Medical Sciences and Research Centre, Hyderabad, Telangana, India. ${ }^{3}$ Department of ENT, Kasturba Medical College, Manipal, Karnataka, India. Email: rakeshvuppala@gmail.com

Received: 09 June 2016, Revised and Accepted: 19 June 2016

\section{ABSTRACT}

Objective: Identifying the role of nasopharyngeal bacteria in recurrent middle ear (ME) infection helps in developing an effective treatment strategy. We aimed to compare bacterial flora of ME with nasopharynx (NP) and their sensitivity patterns in active stage of mucosal disease - otitis media (CSOM).

Methods: Ear and nasopharyngeal swabs from patients with active ear discharge for $>3$ months were processed for culture and sensitivity, the organisms were identified using Gram-stain.

Results: Twenty patients between 10 and 65 years and male:female ratio of 13:7 were enrolled. 9 had symptoms since childhood. 10 patients complained unilateral ear discharge; five each had discharge from the left and right ear. 11 patients had nasal symptoms; nose block due to obstruction (9), nasal discharge (8), and both symptoms were seen in eight patients. 14 patients (70.0\%) had deviated nasal septum and 8 (40.0\%) had discharge in the NP. Of 20 ear swabs, 19 grew bacteria; 1 was sterile (5.0\%). 13 (65\%) nasopharyngeal swabs grew bacteria, 7 were sterile. Culture yielded aerobic bacteria. Staphylococcus aureus was the most common bacterium (9/20, 45.0\%) followed by Pseudomonas (7/20, 35.0\%) isolated from the ear. The most common nasopharyngeal isolate was $S$. aureus $(9=45 \%) .10$ patients had common ME and nasopharyngeal flora. Organisms were sensitive to amoxicillin+clavulanic acid, fluoroquinolones. Sensitivity was observed to polymyxin, trimethoprim, and sulfamethoxazole also.

Conclusion: Common ME and nasopharyngeal flora with same antibiotic sensitivity pattern suggest nasopharyngeal etiology. Nasopharyngeal flora may have a role in recurrent $\mathrm{ME}$ infections in mucosal disease - otitis media.

Keywords: Bacterial flora, Bacterial sensitivity, mucosal disease otitis media, Pseudomonas aeruginosa, Staphylococcus aureus.

(C) 2016 The Authors. Published by Innovare Academic Sciences Pvt Ltd. This is an open access article under the CC BY license (http://creativecommons. org/licenses/by/4. 0/) DOI: http://dx.doi.org/10.22159/ajpcr.2016.v9i5.13366

\section{INTRODUCTION}

Identifying the causative organism and its sensitivity to the antibacterial substance is as important as treatment in chronic otitis media (COM) which is done by culture and sensitivity. Treatment of CSOM is crucial to prevent the recurrence, damage to middle ear (ME) structures, hearing loss and complications. Medical treatment aims at relieving pain, clearing the discharge, reducing disease complications, and removing the causative agent [1]. Treatment goals can be achieved by administering appropriate antibiotics to which the causative organism is sensitive. Caution should be exercised as many of these antibiotics are ototoxic.

One of the complications of CSOM is recurrence. It has been hypothesized that spread of infection from nasopharynx (NP) through Eustachian tube to the ME due to the anatomical connection is one of the causes for recurrence. However, the evidence to support this hypothesis is lacking, particularly in the adult population.

Comparison of ME bacterial flora with that of nasopharyn and their sensitivity patterns can prove useful in identifying the role of nasopharyngeal bacteria in the causation of recurrent ME infection. This can be useful in developing effective treatment strategy, dictating the appropriate topical antibiotic to control the disease activity, and prevent complications.

Mucosal disease - otitis media being common type seen, we conducted this study to identify the bacterial flora of the ME and NP in active stage and evaluated the role of nasopharyngeal flora in ME infections.

\section{METHODS}

This prospective clinical study was done in a tertiary care teaching hospital after obtaining Ethical Committee approval. Patients were screened after obtaining written informed consent, and those met the selection criteria were enrolled.

Patients aged $>10$ years, attending the outpatient department of ear, nose, and throat with history of ear discharge for more than 3 months and with actively discharging ear were included, whereas those who received prior antibiotics in any form either systemic/topical, those with otitis externa, any intra- or extra-temporal suppurative complications, evidence of cholesteatoma, and children with foreign body in the external auditory canal were excluded.

After obtaining a detailed medical history, ear examination under microscopy and nasal endoscopy were done.

Specimen was obtained directly from the ME under microscopic examination using 18 gauge needle bent at $45^{\circ}$ with a plastic cannula attached to $2 \mathrm{ml}$ syringe under aseptic precautions. Nasopharyngeal specimen obtained from the NP around the Eustachian tube orifice, using $5 \mathrm{cc}$ syringe attached to sterile cannula enclosed by sterile catheter passed through the anterior nares to minimize contamination. The tip of the cannula was then pushed through the end of the catheter and specimen aspirated. Before the catheter was removed from the nose, the tip of the cannula was retracted to minimize contamination. This was done under nasal endoscopy. These aspirates were transferred onto the sterile swabs. The culture swabs were immediately sent to the microbiological laboratory. 
The delay between the time of specimen collection and time of inoculation was minimized to $<1 \mathrm{hr}$; if the delay is more than $1 \mathrm{hr}$ but $<2 \mathrm{hrs}$, the specimen tubes were kept in a refrigerator.

Ear and nasopharyngeal swabs were processed immediately for culture and sensitivity, and the organisms were identified using Gram-stain.

After identifying the bacterium, antibiotic sensitivity tests were done. Inhibition zones were compared with standard chart issued by Centre for Disease Control, and if zone of inhibition fell in the range recommended, it is inferred that the bacterium is sensitive to that antibiotic. If the zone of inhibition was not big enough or there was no zone of inhibition, then it was inferred that the bacterium was resistant to that antibiotic. It was declared sterile if no organisms were grown $48 \mathrm{hrs}$ after incubation on suitable media.

Conservative treatment included a systemic antihistamine, nasal decongestants, and broad-spectrum topical antibiotic. Depending on their culture and sensitivity report, a course of appropriate antibiotic was prescribed.

Total study duration was 6 months.

\section{Statistical analysis}

The data were captured on Microsoft Excel worksheet and analyzed. Analyzed data were expressed as descriptive analysis, frequency, mean \pm standard deviation, percentage and in Tables 1 and 2, Fig. 1.

\section{RESULTS}

Twenty patients between 10 and 65 years with a male:female ratio of 13:7 were enrolled. Seven students, three agriculturists, four business professionals, two employees, and four homemakers were included. 9 patients had symptoms since childhood, one since last 12 years, two since last 10 years, four since last 4-5 years, and four had symptoms $<1$ year (4-8 months).

Ten patients complained unilateral ear discharge, five each from the left and right ear, respectively; remaining 10 patients complained of bilateral ear discharge.

11 patients had nasal symptoms; nine had nose block, eight had nasal discharge, and eight had both symptoms. 14 patients (70.0\%) had deviated nasal septum, 8 (40.0\%) had discharge in the NP.

Nasal discharge was seen in 11 patients and six of these patients had bilateral ear discharge.

All our patients complained of symptoms related to ear and nose.

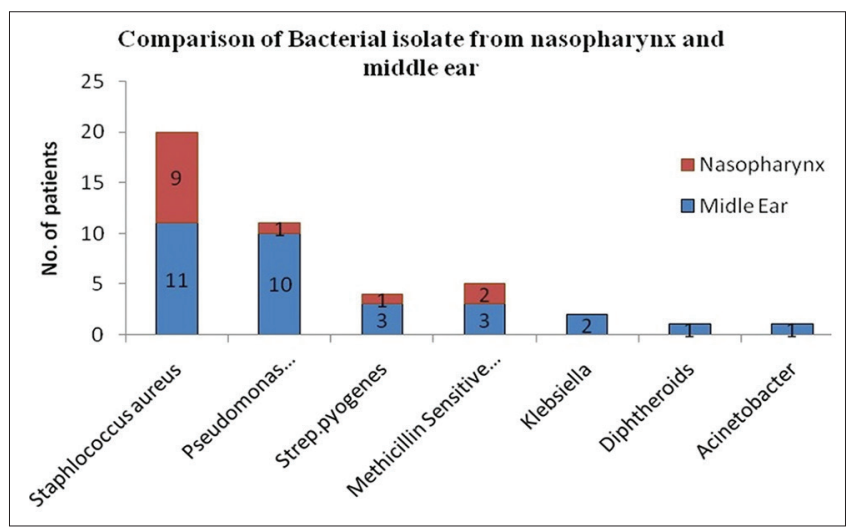

Fig. 1: Comparison the bacterial isolates from nasopharynx and middle ear
Bacterial isolates and bacterial sensitivity

Single isolates (of one genus) were common. Of 20 ear swabs, 19 grew bacteria; one was sterile (5.0\%). 13 (65\%) nasopharyngeal swabs grew bacteria; 7 were sterile.

\section{ME isolates}

Staphylococcus aureus was the most common bacterium (9/20, 45.0\%) isolated from the ear followed by Pseudomonas $(7 / 20,35.0 \%)$ isolated from the ear.

5 (25\%) showed mixed ME microbial flora; 7 (35\%) of NP cultures were sterile after $48 \mathrm{hrs}$ of incubation.

\section{Nasal isolates}

Most common nasopharyngeal isolate is S. aureus ( $9=45 \%) .10$ patients had common ME and nasopharyngeal flora. 8 (80\%) showed S. aureus,

Table 1: Bacterial isolation and bacterial sensitivity-middle ear

\begin{tabular}{|c|c|}
\hline Middle ear & Bacterial sensitivity \\
\hline Organism (n) & Antibiotic (n) \\
\hline P. aeruginosa (4) & $\begin{array}{l}\text { Fluoroquinolones } \\
\text { (ciprofloxacin, ofloxacin, } \\
\text { levofloxacin) (6) }\end{array}$ \\
\hline P. aeruginosa+E. coli (1) & $\begin{array}{l}\text { Polymyxin, trimethoprim, } \\
\text { and sulfamethoxazole (1) }\end{array}$ \\
\hline Strep pyogenes (1) & $\begin{array}{l}\text { Amoxicillin+clavulanic } \\
\text { acid (6) }\end{array}$ \\
\hline Strep pyogenes+MSSA (1) & $\begin{array}{l}\text { Fluoroquinolones } \\
\text { (ciprofloxacin, ofloxacin, } \\
\text { levofloxacin) and } \\
\text { amoxicillin+ } \\
\text { clavulanic acid (3) }\end{array}$ \\
\hline MSSA (7) & $\begin{array}{l}\text { Amoxicillin+clavulanic } \\
\text { acid, trimethoprim and } \\
\text { sulfamethoxazole (1) }\end{array}$ \\
\hline P. aeruginosa+MSSA (1) & Ciprofloxacin (1) \\
\hline K. pneumonia+acinetobacter C (1) & No response (2) \\
\hline \multicolumn{2}{|l|}{ K. pneumonia $+P$ aeruginosa $(1)$} \\
\hline \multicolumn{2}{|l|}{ Coagulase-negative staphylococci (1) } \\
\hline \multicolumn{2}{|l|}{ Diphtheria sps (1) } \\
\hline No organism identified (1) & \\
\hline
\end{tabular}

Table lists the bacterial isolates and antibiotic sensitivity observed in total, not individual correlation. P. aeruginosa: Pseudomonas aeruginosa E. coli: Escherichia Coli, K. pneumonia: Klebsiella pneumonia, MSSA: Methicillinsensitive Staphylococcus aureus

Table 2: Bacterial isolation and bacterial sensitivity- nasopharynx

\begin{tabular}{|c|c|}
\hline Nasopharynx & Sensitivity \\
\hline Organism (n) & Antibiotic (n) \\
\hline P. aeruginosa (1) & $\begin{array}{l}\text { Amoxicillin+clavulanic acid (9) } \\
\text { Fluoroquinolones (ciprofloxacin, ofloxacin, } \\
\text { levofloxacin) (1) }\end{array}$ \\
\hline Strep pyogenes (1) & $\begin{array}{l}\text { Ciprofloxacin (1) } \\
\text { Polymyxin, trimethoprim+sulfamethoxazole } \\
\text { (2) (one pt common in both) }\end{array}$ \\
\hline MSSA (8) & $\begin{array}{l}\text { Polymyxin (1) } \\
\text { Sulfamethoxazole (1) } \\
\text { No response (5) }\end{array}$ \\
\hline $\begin{array}{l}\text { Coagulase-negative } \\
\text { staphylococci (3) } \\
\text { No organism } \\
\text { identified (7) }\end{array}$ & \\
\hline
\end{tabular}

Table lists the bacterial isolates and antibiotic sensitivity observed in total, no individual correlation. MSSA: Methicillin-sensitive Staphylococcus aureus, P. aeruginosa: Pseudomonas aeruginosa 
one Pseudomonas, and one showed Streptococcus pyogenes same flora following common sensitivity patterns was observed in $40 \%$ patients.

Methicillin-sensitive S. aureus (MSSA) was isolated from ME and NP in 2 patients.

\section{Bacterial sensitivity}

Bacterial sensitivity tested showed these organisms were sensitive to amoxicillin+clavulanic acid, fluoroquinolones; sensitivity was observed to polymyxin, trimethoprim, and sulfamethoxazole also (Tables 1 and 2) No sensitivity was seen in two in ME and five in NP cultures.

\section{DISCUSSION}

Chronic otitis media a frequent cause of ear discharge in children, is relatively less common in the adult population. It is classified based on the site of origin of infection, i.e., mucosal disease and atticoantral [2] Although sufficient data is available in the pediatric population, very few studies have been conducted in adult population, and hence, limited data are available.

Identifying the causative organism becomes clinically important, especially in developing countries, where the antibiotics are easily available, and the patient usually would have had a complete or incomplete course of the antibiotic prior visiting the otolaryngologist. As a sequel, there is an increase in the emergence of bacterial resistance and variation in the common disease-causing microbiological flora. Thus, identifying the disease-causing agent and the source of infection becomes crucial in the treatment of CSOM.

We had 20 patients in our study of 6 months; the number of participants in our study is slightly lesser than other studies because of lesser duration and less incidence in the adult population. Moreover, ours being a tertiary care hospital, only those who were unresponsive to treatment or the referred patients received antibiotics.

Male preponderance was observed in our study in line with previous studies [3]. Although we could not establish a relationship between profession and the disease occurrence, more number of students were affected. This could be due to the age factor and the interaction involved in this group.

Unilateral pathology with unilateral complaints was seen in $65 \%$ of patients. Similar observation involvement $(76.36 \%)$ was seen by Kabir et al. and mucosal disease (tubotympanic type) was the most frequent (90\%) [4]. Vishwanath et al. too observed that mucosal disease (tubotympanic) is more frequent (68.1\%) [3].

Of $20 \mathrm{ME}$ samples, only one was sterile. Khanna et al. [5] too have observed sterile cultures in 5\% of patients. Saini et al. [6] observed slightly less culture positivity in adults compared to pediatric population ( $80 \%$ vs. $97.2 \%$ ); however, it was $95 \%$ in our study.

We observed polymicrobial growth in the cultures. Mansoor et al. [7] too noted this in their study in Pakistan, indicating the similarity among the neighboring countries. In contrary, Kabir et al. noted that polymicrobial growth only in $10.91 \%$ of cultures in comparison to pure growth $(79.09 \%)$; sterile culture was in 10\% [4]. Bacterial cultures in our study yielded only aerobes.

Methicillin-resistant S. aureus (45\%) was the most common organism isolated from our patients followed by Pseudomonas aeruginosa (35\%) and coagulase-negative Staphylococcus (5\%). Similar bacterial isolates were noted by Choi et al. [8]. P. aeruginosa and S. aureus were the frequent isolates observed by studies from our country as well as our neighboring countries too [3,7,9-12]. Park et al. [13] too have noted methicillin-resistant $S$. aureus (24.8\%) in their isolates. Various studies have isolated P. aeruginosa [6] and S. aureus from the ME swabs in patients with COM $[5,14,15]$. Loy et al. [16] have observed coagulasenegative Staphylococcus (21.1\%) along with P. aeruginosa (33.3\%) and
S. aureus (33.3\%). Yuen et al. [17] noted that P. aeruginosa (33\%) and S. aureus (15\%) and all the organisms found were aerobes in their study.

Observations by Indudharan et al [18] are similar to Yuen et al. [17] who reported the presence of P. aeruginosa $(27.2 \%)$ and S. aureus $(23.6 \%)$ in their study population. Pseudomonas showed total sensitivity $(100 \%)$ to ciprofloxacin; significant sensitivity $(95.4 \%)$ was shown toward polymyxin B. Significant sensitivity toward ciprofloxacin was noted by Deb and Ray [9].

Supiyaphun et al. [14] have noted sensitivity to ofloxacin and amoxicillin in adult patients with COM. We observed sensitivity to older generation of drugs such as trimethoprim, sulfamethoxazole $(n=1,5 \%)$, in contrast, Park et al. [13] have noted sensitivity in $88.2 \%$ of isolates.

Microbial isolates from ME in our patients were responsive to quinolones namely, ciprofloxacin, ofloxacin, and levofloxacin; there is fast emergence of resistance to these drugs; Jang and Park [19] have documented the emergence of ciprofloxacin-resistant $P$. aeruginosa in their retrospective study.

There have been very few studies which have studied isolates from NP in adult patients with COM [20-22]. Isolates from NP yielded MSSA and coagulase-negative staphylococci in our study. Grewal et al. [20] have isolated staphylococci from the NP in patients with COM. Only 2 patients were positive for MSSA in nasal and ME culture.

Very few studies have correlated the bacterial isolates from ME and NP in adult patients with COM.

Limitation of our study is lesser number of patients analyzed; hence, definite conclusion could not be drawn which could be applied to the larger population; not doing fungal culture is another limitation; we could not do it because of the time constraint in the treatment as ours are mostly referred patients.

\section{CONCLUSION}

The organisms most frequently isolated were staphylococci and Pseudomonas. Common ME and nasopharyngeal flora with same antibiotic sensitivity pattern suggest nasopharyngeal etiology. Nasopharyngeal flora may have a role in the causation of recurrent ME infections in CSOM - mucosal disease.

\section{ACKNOWLEDGMENT}

We acknowledge all patients who willingly participated in our study. We thank and acknowledge the support of Department of Microbiology, Kasturba Medical College, Manipal, in microbiological investigations. We thank the staff of Department of ENT who assisted us during the conduct of the study. We thank Dr. M S Latha, for her support in the preparation and editing of the manuscript.

\section{REFERENCES}

1. Acuin J. Chronic suppurative otitis media. BMJ Clin Evid 2007;2007. pii:0507.

2. Vaidya K, Madhup SK, Shreshta BL, Gautam A, Tuladhar NR. Bacteriological and mycological profile of chronic suppurative otitis media among patients visiting Dhulikhel hospital. ACCLM $2015 ; 1: 37-41$

3. Vishwanath S, Mukhopadhyay C, Prakash R, Pillai S, Pujary K, Pujary P. Chronic suppurative otitis media: Optimizing initial antibiotic therapy in a tertiary care setup. Indian J Otolaryngol Head Neck Surg 2012;64(3):285-9.

4. Kabir MS, Joarder AH, Ekramuddaula FM, Uddin MM, Islam MR, Habib MA. Pattern of chronic suppurative otitis media. Mymensingh Med J 2012;21(2):270-5.

5. Khanna V, Chander J, Nagarkar NM, Dass A. Clinicomicrobiologic evaluation of active tubotympanic type chronic suppurative otitis media. J Otolaryngol 2000;29(3):148-53.

6. Saini S, Gupta N, Aparna, Seema, Sachdeva OP. Bacteriological study 
of paediatric and adult chronic suppurative otitis media. Indian J Pathol Microbiol 2005;48(3):413-6.

7. Mansoor T, Musani MA, Khalid G, Kamal M. Pseudomonas aeruginosa in chronic suppurative otitis media: Sensitivity spectrum against various antibiotics in Karachi. J Ayub Med Coll Abbottabad 2009;21(2):120-3.

8. Choi HG, Park KH, Park SN, Jun BC, Lee DH, Yeo SW. The appropriate medical management of methicillin-resistant Staphylococcus aureus in chronic suppurative otitis media. Acta Otolaryngol 2010;130(1):42-6.

9. Deb T, Ray D. A study of the bacteriological profile of chronic suppurative otitis media in agartala. Indian J Otolaryngol Head Neck Surg 2012;64(4):326-9.

10. Prakash R, Juyal D, Negi V, Pal S, Adekhandi S, Sharma M, et al. Microbiology of chronic suppurative otitis media in a tertiary care setup of uttarakhand state, India. N Am J Med Sci 2013;5(4):282-7.

11. Dayasena R, Dayasiri M, Jayasuriya C, Perera D. Aetiological agents in chronic suppurative otitis media in Sri Lanka. Australas Med J 2011;4(2):101-4.

12. Sharma S, Rehan HS, Goyal A, Jha AK, Upadhyaya S, Mishra SC. Bacteriological profile in chronic suppurative otitis media in Eastern Nepal. Trop Doct 2004;34(2):102-4

13. Park DC, Lee SK, Cha CI, Lee SO, Lee MS, Yeo SG. Antimicrobial resistance of Staphylococcus from otorrhea in chronic suppurative otitis media and comparison with results of all isolated Staphylococci. Eur J Clin Microbiol Infect Dis 2008;27(7):571-7.

14. Supiyaphun P, Kerekhanjanarong V, Koranasophonepun J, Sastarasadhit V. Comparison of ofloxacin otic solution with oral amoxycillin plus chloramphenicol ear drop in treatment of chronic suppurative otitis media with acute exacerbation. J Med Assoc Tha 2000;83(1):61-8.

15. Zivkovic-Marinkov E, Stankovic M, Mihailovic D, Bojanovic M Correlation between histomorphometric changes and the type of aerobic bacteria isolated in chronic suppurative otitis media. Vojnosanit Pregl 2011;68(1):46-50

16. Loy AH, Tan AL, Lu PK. Microbiology of chronic suppurative otitis media in Singapore. Singapore Med J 2002;43(1):296-9.

17. Yuen AP, Chau PY, Wei WI. Bacteriology of chronic suppurative otitis media: Ofloxacin susceptibility. J Otolaryngol 1995;24(3):206-8.

18. Indudharan R, Haq JA, Aiyar S. Antibiotics in chronic suppurative otitis media: A bacteriologic study. Ann Otol Rhinol Laryngol 1999;108(5):440-5.

19. Jang CH, Park SY. Emergence of ciprofloxacin-resistant Pseudomonas in chronic suppurative otitis media. Clin Otolaryngol Allied Sci 2004;29(4):321-3.

20. Grewal DS, Hiranandani NL, Pusalkar AG. The middle ear mucosa in chronic suppurative otitis media. Indian J Otolaryngol 1982;34:1-5.

21. Chang J, Lee SH, Choi J, Im GJ, Jung HH. Nasopharynx as a microbiologic reservoir in chronic suppurative otitis media: Preliminary study. Clin Exp Otorhinolaryngol 2011;4(3):122-5.

22. Afolabi OA, Ologe FE, Nwabuisi C, Salaudeen AG, Ajiboye OT, Nwawolo CC. Correlation of bacterial isolates from middle ear and nasopharynx in patients with chronic suppurative otitis media in Ilorin Nigeria. Malays J Med Sci 2015;22:23-30. 